COMMENT. The syndrome of septo-optic dysplasia appears to be a mild form of holoprosencephaly with single cerebral ventricle and agenesis of the corpus callosum, among other midline defects. Anterior pituitary deficiency is a frequent feature of the syndrome, whereas posterior pituitary disorders are less well documented. In the present study, diabetes insipidus is shown to be a relatively common complication.

\title{
CONGENITAL MIDLINE DEFECT IN PITUITARY DWARFS
}

MRI evaluations of pituitary volume, and clinical and endocrine findings in 101 pituitary dwarfs with congenital idiopathic growth hormone deficiency (CIGHD) are reported from the Departments of Neuroradiology and Pediatrics, Scientific Institute H San Raffaele, Milan, Italy. Ectopia of the posterior pituitary (PPE) was discovered in 59 patients and pituitary volume was reduced. Pituitary hormone deficiency, breech delivery, and other congenital brain anomalies occurred more frequently in PPE patients than in the 42 with normal posterior pituitary except for a narrowed stalk. Associated anomalies included septo-optic dysplasia, with septum pellucidum agenesis and/or hypoplastic optic chiasm, corpus callosum dysgenesis, and basilar impression. A congenital defect involving the pituitary and hypothalamus would account for the MRI abnormalities and the clinico-endocrinological features of CIGHD patients. Breech delivery is the result of the midline brain anomaly, rather than the cause. The hypothesis of a perinatal traumatic transection of the pituitary stalk is contradicted by the findings in this study. (Triulzi $\mathrm{F}$ et al. Evidence of a congenital midline brain anomaly in pituitary dwarfs: a magnetic resonance imaging study in 101 patients. Pediatrics March 1994;93:409-416). (Reprints: Dr Fabio Triulzi, Dept of Neuroradiology, Scientific Institute H S Raffaele, via Olgettina 60, 20132 Milano, Italy).

COMMENT. Major brain midline anomalies, including holoprosencephaly, corpus callosum dysgenesis, and septo-optic dysplasia may be associated with hypothalamo-hypophyseal deficiency. Pituitary gland hypoplasia and ectopia, demonstrated by MRI in this and other studies of CIGHD patients, is not correlated with breech delivery, but is related to an anatomical defect in hypothalamic-pituitary structures.

\section{HYDROCEPHALUS IN OSTEOGENESIS IMPERFECTA}

The neurological complications of osteogenesis imperfecta in 76 patients are reported from the Human Genetics Branch, National Institute of Child Health and Human Development, NIH, Bethesda, MD. The mean age was 8 years. Communicating hydrocephalus was diagnosed by MRI in 17 patients, macrocephaly in 11 , and basilar invagination in 8 , with brainstem compression in 3 . Seizures occurred in 5 patients, and skull fracture in 10 . The importance of detection and treatment of neurological features of osteogenesis imperfecta is noted. (Charnas LR, Marini JC. Communicating hydrocephalus, basilar invagination, and other neurologic features in osteogenesis

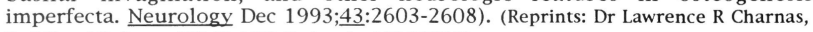
Building 10, Room 9S242, NIH, Bethesda, MD 20892).

COMMENT. The high frequency of basilar impression in severe cases of osteogenesis imperfecta (OI) was remarkable, in comparison with previous reports. Cervical syringohydromyelia is sometimes a concomitant abnormality with basilar impression. 
Mosaic rarefaction of the parietal and occipital bones, a characteristic finding in OI in infancy, may persist throughout childhood, and these strips and linear streaks of diminished density of the calvarium must be distinguished from multiple skull fractures, a common complication of OI. (Caffey J. Pediatric X-Ray Diagnosis, Chicago, Year Book Publ, 1956).

\section{INTELLIGENCE OUTCOME IN SHUNTED HYDROCEPHALUS}

The intelligence outcome of 44 children, tested between 2 and 17 years, and having a shunted hydrocephalus without tumor and with normal ventricular size, was evaluated at the Departments of Child Neurology and Neurosurgery, Instituto Nazionale Neurologico, Milan, Italy. IQ scores ranged from normal to highly defective. Verbal IQs were always higher than performance IQs. Variables without effect on IQ were: 1) site of obstruction; 2) number of shunt revisions; and 3) history of seizures. Verbal IQ was influenced negatively by antiepileptic therapy and motor deficits, and positively by an older age at time of shunting. Non-verbal, performance IQ was lower in patients with cerebral hemisphere malformations, those with more than one shunt, pre- and perinatal problems, and antiepileptic therapy. The side of shunt placement was significantly correlated with non-verbal IQ insertion on the right ensuring a better outcome. Posterior fossa malformations were not correlated with IQ. (Riva D et al. Intelligence outcome in children with shunted hydrocephalus of different etiology. Child's Nerv Syst Jan 1994;10:70-73). (Respond: Dr Daria Riva, Dept of Child Neurology, Instituto Nazionale Neurologico "C Besta", Via Celoria, 11, I-20133 Milan, Italy).

COMMENT. Shunted hydrocephalic children have a preferential loss of non-verbal IQ, Verbal and Performance IQs are influenced by different factors. Verbal IQ is correlated mainly with antiepileptic therapy, while non-verbal IQ was dependent on several surgical and medical variables.

A study of long-term outcome of hydrocephalus at the Service de Neurochirurgie Pediatrique, Hopital Necker-Enfants Malades, Paris, France, showed that IQ was related more to etiology than to ventricular dilatation, and to time of treatment. IQs are higher in patients with meningomyeloceles than in those with brain parenchymal lesions, toxoplasmosis, hemorrhage, or meningitis. IQs were above 80 in $60 \%$ of those shunted before 2 months of age and in only $29 \%$ treated after 2 years. (Hirsch J-F. Consensus: long-term outcome in hydrocephalus. Child's Nerv Syst Jan 1994;10:64-69).

A unifying theory for the definition and classification of hydrocephalus is proposed by Raimondi AJ, University of Rome. (Child's Nerv Syst Jan 1994;10:2-12). Hydrocephalus is a pathological increase in intracranial CSF volume, either 1) intraparenchymal (cerebral edema), or 2) extraparenchymal (subarachnoid, cisternal, or intraventricular), and independent of hydrostatic or barometric pressure. Ventricular or subarachnoid dilatation occurs as a result of intermittent increases in extraparenchymal CSF volume. Hydrocephalus may be present in a child who does not yet have dilated ventricles but in whom both CSF volume and pressure are increased. Cerebral edema may cause the same volumetric changes as increases in intraventricular fluid volume, and the term internal hydrocephalus is of little significance. 